\title{
Association between gastrointestinal symptoms and disease severity in patients with COVID-19 in Tehran City, Iran
}

\author{
Elena Lak ${ }^{1}$, Seyed Amir Sheikholeslami ${ }^{2}$, Mahmoud Dehghani Ghorbi ${ }^{3}$, Mostafa Shafei ${ }^{4}$, Homayon Yosefi ${ }^{5}$ \\ ${ }^{1}$ Department of Gastroenterology, School of Medicine, Imam Hossein Hospital Shahid Beheshti University of Medical Science, \\ Tehran, Iran \\ 2Department of Adult Haematology and Oncology, School of Medicine, Imam Hossein Hospital, Shahid Beheshti University \\ of Medical Sciences, Tehran, Iran \\ ${ }^{3}$ Department of Haematology and Oncology, School of Medicine, Imam Hossein Hospital, Shahid Beheshti University \\ of Medical Sciences, Tehran, Iran \\ ${ }^{4}$ School of Health, Ahvaz Jundishapur University of Medical Sciences, Ahvaz, Iran \\ ${ }^{5}$ Department of Oncology, School of Medicine, Ahvaz Jundishapur University of Medical Sciences, Ahvaz, Iran
}

Gastroenterology Rev 2022; 17 (1): 52-58

DOI: https://doi.org/10.5114/pg.2021.106245

Key words: gastrointestinal symptom, COVID-19, Iran.

Address for correspondence: Homayon Yosefi, Department of Oncology, School of Medicine, Ahvaz Jundishapur University of Medical Sciences, Ahvaz, Iran, e-mail: epidemiology2021@gmail.com

\begin{abstract}
Introduction: It is important to identify the relationship between COVID-19 and gastrointestinal symptoms for health organizations in different communities.

Aim: To assess the relationship between gastrointestinal symptoms and COVID-19.

Material and methods: It was a cross-sectional descriptive-analytical study that was conducted on 381 patients those where admitted to Imam Hossein Hospital with a confirmed diagnosis of COVID-19 on nasopharyngeal polymerase chain reaction testing for SARS-CoV-2 from first March to end of June in Tehran city; 2020. Data was entered and analyzed by using SPSS version 22 and level of significant was consider less than 0.05.

Results: Out of all the patients with COVID-19, 164 (43\%) had gastrointestinal symptoms. The most symptoms of them were nausea (19.2\%), vomiting (17.2\%), abdominal pain (15.7\%), diarrhoea (12.6\%), haematomas (1\%), melena (1.6\%), rectal bleeding (1.6\%), and constipation (1.8\%), respectively. The mean D-dimer1 value was significantly different between the 2 groups with gastrointestinal symptoms and no gastrointestinal symptoms. In other words, there is a strong relationship between the variable D-dimer1, which is one of the important symptoms showing the severity of COVID-19 disease, and gastrointestinal symptoms $(p<0.0001)$.

Conclusions: Our finding shows a statistical relationship between the level of D-dimer and gastrointestinal symptoms in patients with COVID-19. The mortality rate was higher in patients with gastrointestinal symptoms, which is an important outcome for gastroenterologists.
\end{abstract}

\section{Introduction}

A novel coronavirus has spread throughout China, originating from the city of Wuhan, and has caused many deaths so far [1]. It is a highly contagious virus that has spread rapidly and efficiently. Coronavirus disease 2019 (COVID-19) is caused by a virus (SARS-CoV-2) from the same family as the lethal coronaviruses that caused severe acute respiratory syndrome (SARS-CoV) and Middle East respiratory syndrome (MERS-CoV). COVID-19 is a relatively large virus (120 nm) and is enveloped, containing a positive-sense single-stranded RNA [2]. The virus is transmitted through direct contact with the infected person's respiratory droplets (coughing and sneezing), as well as contact with infected surfaces. The COVID-19 virus can survive for days on surfaces, but a simple disinfectant can eliminate this [3]. COVID-19 signs and symptoms include fever, cough, and 
shortness of breath. In more severe cases, infection can lead to pneumonia, serious respiratory problems, and, ultimately, fatalities. Thousands of people have been reported to have been infected with the virus so far [4]. Respiratory symptoms are the main symptoms, but gastrointestinal symptoms such as diarrhoea, nausea, and vomiting are also among the primary symptoms $[5,6]$.

The virus is excreted in the faeces, which is a sign of gastrointestinal involvement. The most common gastrointestinal symptoms are; anorexia, diarrhoea, nausea, and vomiting. A stool exam may still be positive after the breath sample is negative [7]. Patients with gastrointestinal symptoms may not have typical coronary symptoms [8].

Occurrence of Gl symptoms is due to the intestinal tropism of COVID-19 [9]. Moreover, GI symptoms can coexist or even precede respiratory manifestations [10]. Rarely, COVID-19 patients can present with only GI symptoms, without respiratory symptoms [11].

The relationship between many gastrointestinal, clinical, and laboratory parameters and disease severity in different communities is not yet fully known. Examining more parameters can help improve the accuracy of anticipation and prognosis of the disease. On the other hand, patients with critical conditions should be hospitalized in the intensive care unit, while mild patients can be kept and treated separately. Also, due to the widespread outbreak of this virus and the high number of patients, it is necessary to identify the determinant indicators of patients who are the highest risk group for the severity of the disease [12].

After the outbreak of COVID-19 disease in different countries of the world, many studies have been conducted abroad on the subject of the gastrointestinal tract [13-15], but no study has been done on this case inside the country.

\section{Aim}

Therefore, the aim of this study was to assess the relationship between gastrointestinal symptoms and disease severity in patients with COVID-19.

\section{Material and methods}

This was a cross-sectional descriptive-analytical study that was conducted on 381 patients who were admitted to Imam Hossein Hospital with a confirmed diagnosis of COVID-19 on nasopharyngeal polymerase chain reaction testing for SARS-CoV-2 from 1 March to the end of June 2020 in Tehran city.

\section{Exclusion criteria}

Patients were excluded if they were younger than 18 years, were not hospitalized and managed on an am- bulatory basis, were pregnant, or for whom results of SARS-CoV-2 nasopharyngeal testing were unavailable.

\section{Study tool}

A checklist was made by researchers, which includes 5 sections: demographic information; general symptoms including asthma, cough, fever, chills, fatigue, loss sense of smell, etc.; gastrointestinal symptoms including nausea, vomiting, dark stools, constipation, diarrhoea, etc.; laboratory results; and the final consequences of survival or death. If a particular laboratory test was not performed at the time of admission, the first laboratory values within $24 \mathrm{~h}$ of the admission were used.

\section{Ethical considerations}

This study was supported by the Deputy of Research in Shahid Beheshti University of Medical Sciences with reference No. IR.SBMU.RETECH.REC.1399.090, and all subjects gave informed consent before participation in this study.

\section{Statistical analysis}

Statistical analysis was performed by using SPSS software version 22 (SPSS Inc., Armonk, NY, USA). The Kolmogorov-Smirnov test was used to evaluate the normality of data distribution in 2 groups: with gastrointestinal symptoms and without gastrointestinal symptoms. Descriptive summary statistics are presented as means and SD for continuous variables and frequencies with percentages for categorical variables. Categorical and continuous variables were tested for statistical significance using $\chi^{2}$ tests and $t$-tests, respectively. For continuous variables that were not normally distributed, we utilized the nonparametric test such as the Mann-Whitney $U$ test to compare the groups. The significance level was considered at less than 0.05 .

\section{Results}

\section{Outbreak rate of gastrointestinal symptoms in COVID-19 patients}

A total of 381 patients were hospitalized with confirmed COVID-19 during the study period. Female and male patients comprised 147 (38.6\%) and 234 (61.4\%), respectively.

The mean age was $62.6 \pm 17.1$ years. Of these, 164 (43\%) patients had gastrointestinal symptoms, and 217 (57\%) did not.

The most common gastrointestinal symptoms in COVID-19 patients were nausea, vomiting, abdominal pain, and diarrhoea. $19.2 \%$ of patients had nausea, and this gastrointestinal symptom was more common in 
Table I. Frequency of gastrointestinal symptoms

\begin{tabular}{lc} 
Gastrointestinal symptoms & Frequency (\%) \\
\hline Hematemesis: & $377(99)$ \\
\hline No & $4(1)$ \\
\hline Yes & $375(98.4)$ \\
\hline Melena: & $6(1.6)$ \\
\hline No & \\
\hline Yes & $375(98.4)$ \\
\hline Rectorrhagia: & $6(1.6)$ \\
\hline No & $308(80.8)$ \\
\hline Yes & $73(19.2)$ \\
\hline Nausea: & $315(82.7)$ \\
\hline No & $66(17.3)$ \\
\hline Yes & $333(87.4)$ \\
\hline Vomiting: & $48(12.6)$ \\
\hline No & $374(98.2)$ \\
\hline Yes & \\
\hline Constipation: & \\
\hline No & \\
\hline Yes & \\
\hline Diarrhoea: & \\
\hline No & \\
\hline Yes & \\
\hline Abdominal sonography: & \\
\hline Nos & \\
\hline
\end{tabular}

women than men (male and female $15.4 \%$ and $25.2 \%$, respectively). Also $17.2 \%$ of patients had vomiting, and this symptom was observed much more in women than in men. $15.7 \%$ of patients had abdominal pain, which was also much more common in women than in men. Diarrhoea occurred in $13.7 \%$ of male and $10.9 \%$ of females.

Four patients had haematemesis (1\%), and melena was found in $6(1.6 \%)$ patients. Other features of the sample under review are listed in Table I.

There was no statistical difference between the 2 groups in values of laboratory data such as mean haemoglobin, white blood cells (WBC), lymphocyte, and platelet counts. The mean ferritin level was lower in the cases than in the controls but did not reach statistical significance (777 vs. $951 \mathrm{ng} / \mathrm{ml}, p=0.61$ ). Mean C-reactive protein (CRP), creatinine, and lactic acid levels were higher but not statistically significant in both groups, as noted in.

For the whole group of subjects, the mean levels of laboratory data at the time of admission were as follows: WBC 12.1; creatinine (Cr) 2.12; urea 53.7; aspartate aminotransferease (AST) 61.9; alkaline phosphatase (ALP) 216.4; mean lactate dyhydrogenase (LDH) 630.6; mean D-dimer 2247.1; CRP 56.3, and international normalized ratio (INR) 1.31. The means of these variables in the gender groups of men and women as well as in the groups with gastrointestinal symptoms and no symptoms are given in Table II.

\section{Relationship between age, gender, and body mass index (BMI) of people with and without gastrointestinal symptoms}

The 2-sample independent $t$-test was used to evaluate the relationship between age of COVID-19 patients and gastrointestinal symptoms.

Table II. Mean laboratory data levels at the time of admission

\begin{tabular}{lcccccc} 
Variable & Male & Female & $P$-value & $\begin{array}{c}\text { CovID-19 patient with } \\
\text { gastrointestinal symptoms } \\
\text { (mean) }\end{array}$ & $\begin{array}{c}\text { CovID-19 patient without } \\
\text { gastrointestinal symptoms } \\
\text { (mean) }\end{array}$ & $P$-value \\
\hline White blood cell & 14.81 & 8.0095 & 0.36 & 9.0659 & 14.544 & 0.46 \\
\hline Creatinine & 2.1762 & 2.0317 & 0.754 & 2.7337 & 1.657 & 0.03 \\
\hline Urea & 56.756 & 49.037 & 0.142 & 62.596 & 45.543 & 0.001 \\
\hline Aspartate transaminase & 72.108 & 45.087 & 0.074 & 80.94 & 47.025 & 0.102 \\
\hline Alkalosis & 211.76 & 224.41 & 0.553 & 240.98 & 197.18 & 0.047 \\
\hline Lactic dehydrogenase & 662.48 & 575.99 & 0.401 & 669.58 & 594.47 & 0.46 \\
\hline D-dimer & 2405.8 & 1991.9 & 0.560 & 3651.4 & 60.422 & $<0.0001$ \\
\hline Cyclic receptor protein & 60.81 & 49.25 & 0.053 & 50.932 & 1.3398 & 0.103 \\
\hline International normalized ratio & 1.2476 & 1.4084 & 0.466 & 1.275 & & 0.765
\end{tabular}


The mean age of patients in the 2 groups was not statistically significant $(p=0.317)$. The $\chi^{2}$ test was used to examine the relationship between gender and gastrointestinal symptoms in patients with COVID-19, showing that there was statistically significant between gender and gastrointestinal symptoms ( $p<0.0001)$. Also, there was no statistically significant difference between the BMI of patients and gastrointestinal symptoms $(p=0.35)$ (Table III).

\section{Relationship between age and BMI and length of hospital stay with outcome (death or improved)}

In the group of improved patients, the mean age was 60.2 years, with a standard deviation of 16.8 years and with an age range of 20 to 94 years.

In the death group, the mean age was 72.9 years with a standard deviation of 14.7 years with an age range of 41 to 100 years. There was a statistically significant difference between the 2 groups in terms of age $(p<0.001)$.

The mean body mass index was $28.4 \pm 12.2$ in the improved group and $26.84 \pm 8.7$ in the deceased group, which was not statistically significant between the 2 groups $(p>0.05)$. The mean number of hospitalization days in the hospital in the recovered patient group was 6.76 days and in the deceased group it was 7.06 days. In other words, the length of stay of the deceased patients was 0.3 days longer than the improved patients, but this difference was not statistically significant $(p=0.493>0.05)$ (Table IV).

\section{Relationship between gastrointestinal symptoms and severity of COVID-19 disease}

The 2-sample independent $t$-test was used to investigate the relationship between the variables of severity of COVID-19 disease and gastrointestinal symptoms. The severity of COVID-19 disease was assessed in 2 groups: patients with gastrointestinal symptoms and patients without gastrointestinal symptoms. The results are shown in Table V.

According to the 2-sample independent $t$-test, only the mean D-dimer1 level was significantly different between the 2 groups (with gastrointestinal symptoms and without gastrointestinal symptoms). In other words, there is a strong relationship between the D-dimer 1 variable, which is one of the important symptoms showing the severity of COVID-19 disease, and gastrointestinal symptoms $(p<0.0001)$. As can be observed in Table V, the mean D-dimer1 value is 3651 in the group with no gastrointestinal symptoms and 933 in the group without gastrointestinal symptoms, which indicates a significant difference between the 2 groups, but other severity of COVID-19 disease.

According to the above table, among LDH, erythrocyte sedimentation rate (ESR), D-dimer and $\mathrm{O}_{2}$ PulseOximeter variables, D-Dimer variable had a significant difference between the two groups of patients with gastrointestinal symptoms and without gastrointestinal symptoms. The means of ESR, CRP, dimer, LDH, and $\mathrm{O}_{2}$ PulseOximeter are also seen in the 2 groups in Table V.

Table III. Relationship between age, gender, and BMI of people with and without gastrointestinal symptoms

\begin{tabular}{lccc} 
Characteristics & $\begin{array}{c}\text { COVID-19 patients with } \\
\text { gastrointestinal symptoms }\end{array}$ & $\begin{array}{c}\text { COVID-19 patients without } \\
\text { gastrointestinal symptoms }\end{array}$ & $P$-value \\
\hline Age, mean (SD) & $63.66(17.195)$ & $61.88(17.123)$ & 0.31 \\
\hline BMI, mean (SD) & $29.98(16.47)$ & $27.5(6.39)$ & 0.35 \\
\hline Female gender, $n(\%)$ & $78(47.6)$ & $69(31.8)$ & $<0.0001$
\end{tabular}

Table IV. Relationship between age, BMI, and length of hospital stay with outcome

\begin{tabular}{|c|c|c|c|c|c|}
\hline Variable & Outcome & Frequency & Mean & Standard deviation & $P$-value \\
\hline \multirow[t]{2}{*}{ Age } & Improved & 307 & 60.19 & 16.8 & $<0.001$ \\
\hline & Death & 74 & 72.86 & 14.74 & \\
\hline \multirow[t]{2}{*}{ BMI } & Improved & 239 & 28.35 & 12.21 & 0.207 \\
\hline & Death & 43 & 26.84 & 8.7 & \\
\hline \multirow{2}{*}{$\begin{array}{l}\text { Duration of hospital } \\
\text { stay }\end{array}$} & improved & 307 & 6.76 & 5.32 & 0.493 \\
\hline & death & 74 & 7.06 & 6.89 & \\
\hline
\end{tabular}


Table V. Relationship between gastrointestinal symptoms and severity of COVID-19 disease

\begin{tabular}{|c|c|c|c|c|c|}
\hline Variable & Groups & Frequency & Mean & SD & $P$-value \\
\hline \multirow{2}{*}{$\begin{array}{l}\text { Cyclic receptor } \\
\text { protein }\end{array}$} & Gastrointestinal symptoms & 157 & 50.93 & 52.80 & 0.107 \\
\hline & No gastrointestinal symptoms & 204 & 60.42 & 57.17 & \\
\hline \multirow[t]{2}{*}{ ESR } & Gastrointestinal symptoms & 126 & 45.45 & 26.74 & 0.551 \\
\hline & No gastrointestinal symptoms & 145 & 47.35 & 25.46 & \\
\hline \multirow{2}{*}{$\begin{array}{l}\text { Lactic } \\
\text { dehydrogenase }\end{array}$} & Gastrointestinal symptoms & 93 & 669.58 & 950.16 & 0.45 \\
\hline & No gastrointestinal symptoms & 100 & 594.47 & 275.80 & \\
\hline \multirow[t]{2}{*}{ D-dimer } & Gastrointestinal symptoms & 29 & 3651.44 & 3030.50 & $<0.0001$ \\
\hline & No gastrointestinal symptoms & 31 & 933.46 & 1219.79 & \\
\hline \multirow{2}{*}{$\begin{array}{l}\mathrm{O}_{2} \text { pulse } \\
\text { oximeter }\end{array}$} & Gastrointestinal symptoms & 114 & 89.82 & 2.46 & 0.559 \\
\hline & No gastrointestinal symptoms & 168 & 89.24 & 2.32 & \\
\hline
\end{tabular}

Table VI. Relationship between severity of COVID-19 disease and gastrointestinal symptoms according to qualitative variables

\begin{tabular}{|c|c|c|c|c|}
\hline Variable & $\begin{array}{l}\text { With gastrointestinal symptoms } \\
\qquad n(\%)\end{array}$ & $\begin{array}{l}\text { Without gastrointestinal symptoms } \\
\qquad n(\%)\end{array}$ & Total & $P$-value \\
\hline Outcome: & & & & 0.41 \\
\hline Improved & $307(80.6)$ & $178(82)$ & $129(78.7)$ & \\
\hline Death & $74(19.4)$ & $39(18)$ & $35(21.3)$ & \\
\hline Unilateral lung involvement: & & & & 0.526 \\
\hline Yes & $42(11)$ & $22(10.1)$ & $20(12.2)$ & \\
\hline No & $339(89)$ & $195(89.9)$ & $144(87.8)$ & \\
\hline Bilateral lung involvement: & & & & 0.589 \\
\hline Yes & $294(87.5)$ & $167(88.4)$ & $127(86.4)$ & \\
\hline No & $42(12.5)$ & $22(11.6)$ & $20(13.6)$ & \\
\hline
\end{tabular}

The $\chi^{2}$ test was used to evaluate the relationship between mortality frequency and pulmonary involvement rate with or without gastrointestinal symptoms. One of the most important symptoms in diagnosing the severity of COVID-19 disease is the frequency of death in the groups.

In the study of pulmonary involvement rate in patients, no statistically significant relationship was observed between the severity of COVID-19 disease and the rate of pulmonary involvement in these patients $(p=0.589)$ (Table VI).

\section{Discussion}

In this study, we found that 164 (43\%) of the patients hospitalized with COVID-19 presented with at least one gastrointestinal (GI) symptom such as diar- rhoea, nausea, vomiting, or abdominal pain. Nausea was the most common Gl symptom, followed by vomiting, abdominal pain, and diarrhoea. There were no significant differences in terms of patient demographics between patients with and without Gl symptoms, but there were significant differences between some laboratory data such as creatinine, urea and D-dimer between patients with and without GI symptoms. Furthermore, there was no association between severity of COVID-19 disease and gastrointestinal symptoms.

Our study shows that the prevalence of Gl symptoms in COVID-19 patients is $43 \%$, which is lower than the prevalence of Gl symptoms reported by other studies in the USA and China in the range of 50.5-61.3\% $[9,16]$. The higher reported rate of Gl symptoms in those studies could probably be due to the inclusion of 
anorexia as one of the GI symptoms. Anorexia is a nonspecific symptom that could be related to an overall infectious or inflammatory process and hence was not included as a specific GI symptom in our study. In a US study by Redd et al. [17], the prevalence of Gl symptoms in COVID-19 patients was reported to be as high as $61.3 \%$. In that study, anorexia was reported in $34.8 \%$ of cases, diarrhoea in $33.7 \%$, and nausea in $26.4 \%$. In a study by Guan et al. [9] from Wuhan, China, even though about $50 \%$ reported having Gl symptoms, the majority of these patients had anorexia (78.6\%). When anorexia was excluded from the analysis, only $18.6 \%$ had specific GI symptoms. A trend of increasing recognition of GI manifestations among COVID-19 patients has been noted since its outbreak in Wuhan, China. During the original outbreak in Wuhan, diarrhoea was reported in only $3 \%$ of the cases [18]. This number increased to $10 \%$ in a subsequent study from Wuhan and $25 \%$ in a study from Singapore [3, 19]. As awareness is increasing among health-care workers about the Gl manifestations in COVID-19 patients, the reports of the presence of Gl symptoms are increasing in the studies. In our study, there was no association between the GI symptoms and outcomes in COVID-19 patients. Previous studies have reported conflicting findings concerning the presence of Gl symptoms and poor outcomes. In the study by Guan et al. [9] from Wuhan, China, patients with digestive symptoms had longer LOS (9 vs. 7.3 days, $p=0.013)$. Furthermore, this study noted that as the severity and duration of COVID-19 increase, GI symptoms increase as well. In a multicentre study of $191 \mathrm{pa-}$ tients by Zhou et al. [20], the presence of GI symptoms was associated with elevated CRP (7.3 vs. $3.8 \mathrm{mg} / \mathrm{l}$, $p=0.021)$, elevated alanine aminotransferase (64.1 vs. $46.6 \mathrm{U} / \mathrm{l}, p=0.049$ ), and lower haemoglobin levels, when compared to patients without GI symptoms. However, in the study by Redd et al. [17] there were no differences in clinical outcomes in patients with or without GI symptoms. Also, they reported no significant differences in the leukocyte count, haemoglobin, platelets, coagulation, or liver tests in groups with or without Gl symptoms. Although the specific mechanisms causing GI manifestations in COVID-19 are not entirely known, there are several proposed theories. Intestinal tropism has been noted with SARS-CoV-2, which could be due to its strong affinity to angiotensin-converting enzyme-2 receptors, and angiotensin-converting enzyme-2 receptors are highly expressed in the oesophagus and intestinal epithelial cells [21]. Hence, there is a strong possibility of direct small bowel involvement, resulting in direct cytopathic effects causing Gl symptoms. Furthermore, Redd et al. [17] noted that loss of smell (anosmia) and loss of taste (ageusia) were commonly associated with nausea (adjusted OR $=2.71,95 \% \mathrm{Cl}$ : $1.21-6.20 ; p=0.015$ ) and anorexia (adjusted $\mathrm{OR}=3.70$, 95\% Cl: $1.49-9.16 ; p=0.0048$ ) after controlling for potential confounders. While the exact cause of this association is unclear, it could be due to damage to olfactory and gustatory receptors during viral entry through nasal and oral routes [22]. Additionally, in a study from Hong Kong, patients with diarrhoea on presentation had higher rates of stool RNA positivity when compared to those without diarrhoea (38.5 vs. $8.7 \%, p=0.02$ ). This is suggestive of the direct effects of the SARS-CoV-2 on the Gl tract [19]. Also, the viral infection can cause altered intestinal permeability, resulting in malabsorption [23]. Finally, the inflammatory response from a cytokine storm in severe COVID-19 patients can cause hypoxia-induced bowel ischaemia and contribute to diarrhoea. Gastrointestinal symptoms were associated with D-dimer1 rate in patients, but other variables such as CRP, ESR, LDH, pulse oximeter, and pulmonary involvement rate did not have a statistically significant relationship with having gastrointestinal symptoms. Despite the lack of a significant relationship between mortality due to COVID-19 disease and gastrointestinal symptoms, a significant difference in the frequency of mortality was observed between the 2 groups (with gastrointestinal symptoms and without symptoms).

Specific limitations to this study include the cross-sectional design, relatively single-centre hospital-based nature, and lack of validated symptom instruments. This could introduce selection bias and limit the reliability and generalizability of the results. We could not correlate the presence of SARS-CoV-2 RNA with Gl symptoms because this test was not routinely performed in our institution.

The second limitation was related to the selection of patients who were hospitalized in the hospital and hence a high frequency of pulmonary involvement in the group of patients who did not have gastrointestinal symptoms. Therefore, the lack of a significant of relationship between pulmonary involvement and gastrointestinal symptoms with the severity of COVID-19 disease may be due to the fact that only patients with moderate to severe disease were referred to hospital. Therefore, it cannot be said with certainty that the rate of pulmonary involvement has no relationship with gastrointestinal symptoms.

\section{Conclusions}

Gl symptoms are commonly encountered in hospitalized COVID-19 patients. In our study the most common gastrointestinal symptom was nausea. Gastrointestinal symptoms of nausea, vomiting, and abdominal pain were more common in women, and diarrhoea was 
more common in men. Gastrointestinal symptoms had a statistically significant relationship with the rate of D-dimer1, and the mean value of D-dimer1 in patients with gastrointestinal symptoms was more than in patients without gastrointestinal symptoms. Also, the incidence of death in patients with gastrointestinal symptoms was more than in patients without gastrointestinal symptoms. Other variables indicating the severity of COVID-19 disease, such as pulmonary involvement rate, did not have significant relationship with gastrointestinal symptoms. The reason for this might be the acute diseases among people who are hospitalized.

\section{Acknowledgments}

We are grateful to all patients for their cooperation.

\section{Conflict of interest}

The authors declare no conflict of interest.

\section{References}

1. Zarei J, Dastoorpoor M, Jamshidnezhad A, et al. Regional COVID-19 registry in Khuzestan, Iran: a study protocol and lessons learned from a pilot implementation. Inform Med Unlocked 2021; 23: 100520.

2. Santarpia JL, Rivera DN, Herrera V, et al. Transmission potential of SARS-CoV-2 in viral shedding observed at the University of Nebraska Medical Center. medRxiv 2020. doi: https://doi.org/ 10.1101/2020.03.23.20039446.

3. World Health Organization. Coronavirus disease 2019 (COVID-19): Situation Report - 43. Available from: https:// www.who.int/docs/default-source/coronaviruse/situation-reports/20200303-sitrep-43-covid-19.pdf?sfvrsn=2c21c09c_2.

4. Xu XW, Wu XX, Jiang XG. Clinical findings in a group of patients infected with the 2019 novel coronavirus (SARS-Cov-2) outside of Wuhan, China: Retrospective case series. BMJ 2020 368: $\mathrm{m} 606$.

5. de Oliveira AP, Lopes ALF, Pacheco G, et al. Premises among SARS-CoV-2, dysbiosis and diarrhea: Walking through the ACE2/mTOR/autophagy route. Med Hypotheses 2020; 144: 110243.

6. Nie K, Yang YY, Deng MZ, Wang XY. Gastrointestinal insights during the COVID-19 epidemic. World J Clin Cases 2020; 8: 3934-41.

7. Galanopoulos M, Gkeros F, Doukatas A, et al. COVID-19 pandemic: pathophysiology and manifestations from the gastrointestinal tract. World J Gastroenterol 2020; 26: 4579-88.

8. Christoph GD, Dolores H, Gernot M, et al. Primary presentation of COVID-19 solely with gastrointestinal symptoms: a problem for the containment of the disease. Eur J Gastroenterol Hepatol 2020; 32: 1475-8.

9. Guan WJ, Ni ZY, Hu Y, et al. Clinical characteristics of coronavirus disease 2019 in China. N Engl J Med 2020; 382: 1708-20.

10. Liang W, Feng Z, Rao S, et al. Diarrhoea may be underestimated: a missing link in 2019 novel coronavirus. Gut 2020; 69: 1141-3.
11. Pan L, Mu M, Yang P, et al. Clinical characteristics of COVID-19 patients with digestive symptoms in Hubei, China: a descriptive, cross-sectional, multicenter study. Am J Gastroenterol 2020; 115: 766-73.

12. Wu G, Zhou S, Wang Y, et al. A prediction model of outcome of SARS-CoV-2 pneumonia based on laboratory findings. Sci Rep 2020; 10: 14042.

13. Henry BM, Oliveira MHS, Benoit S, et al. Hematologic, biochemical and immune biomarker abnormalities associated with severe illness and mortality in coronavirus disease 2019 (COVID-19): a meta-analysis. Clin Chem Lab Med 2020; 58 : 1021-8.

14. Zheng Z, Peng F, Xu B, et al. Risk factors of critical and mortal COVID-19 cases: a systematic literature review and meta-analysis. J Infection 2020; 81 e16-25.

15. Tang N, Li D, Wang X, Sun Z. Abnormal coagulation parameters are associated with poor prognosis in patients with novel coronavirus pneumonia. J Thromb Haemost 2020; 18: 844-7.

16. Han C, Duan C, Zhang S, et al. Digestive symptoms in COVID-19 patients with mild disease severity: clinical presentation, stool viral RNA testing, and outcomes. Am J Gastroenterol 2020; 115: 916-23.

17. Redd WD, Zhou JC, Hathorn KE, et al. Prevalence and characteristics of gastrointestinal symptoms in patients with SARSCoV-2 infection in the United States: a multicenter cohort study. Gastroenterology 2020; S0016-5085(20): 30564-3.

18. Huang C, Wang Y, Li X, et al. Clinical features of patients infected with 2019 novel coronavirus in Wuhan, China. Lancet 2020; 395: 497-506.

19. Cheung KS, Hung IF, Chan PP, et al. Gastrointestinal manifestations of SARS-CoV-2 infection and virus load in fecal samples from the Hong Kong cohort and systematic review and meta-analysis. Gastroenterology 2020; S0016-5085: 30448-0.

20. Zhou F, Yu T, Du R, et al. Clinical course and risk factors for mortality of adult inpatients with COVID-19 in Wuhan, China: a retrospective cohort study. Lancet 2020; 395: 1054-62.

21. Zou X, Chen K, Zou J, et al. Single-cell RNA-seq data analysis on the receptor ACE2 expression reveals the potential risk of different human organs vulnerable to 2019-nCoV infection. Front Med 2020; 14: 185-92.

22. Vaira L, Salzano G, Deiana G, De Riu G. Anosmia and ageusia: common findings in COVID-19 patients. Laryngoscope 2020; 130: 1787.

23. Gu J, Han B, Wang J. COVID-19: gastrointestinal manifestations and potential fecal-oral transmission. Gastroenterology 2020; 158: 1518-9.

Received: 27.03.2021

Accepted: 28.04.2021 